\title{
ORIGINAL ARTICLE Perisomatic changes in h-channels regulate depressive behaviors following chronic unpredictable stress
}

\author{
CS Kim, DH Brager and D Johnston
}

\begin{abstract}
Chronic stress can be a precipitating factor in the onset of depression. Lentiviral-mediated knockdown of HCN1 protein expression and reduction of functional $I_{\mathrm{h}}$ produce antidepressant behavior. However, whether h-channels are altered in an animal model of depression is not known. We found that perisomatic HCN1 protein expression and $I_{\mathrm{h}}$-sensitive physiological measurements were significantly increased in dorsal but not in ventral CA1 region/neurons following chronic unpredictable stress (CUS), a widely accepted model for major depressive disorder. Cell-attached patch clamp recordings confirmed that perisomatic $I_{\mathrm{h}}$ was increased in dorsal CA1 neurons following CUS. Furthermore, when dorsal CA1 $I_{\mathrm{h}}$ was reduced by shRNA-HCN1, the CUS-induced behavioral deficits were prevented. Finally, rats infused in the dorsal CA1 region with thapsigargin, an irreversible inhibitor of the SERCA pump, exhibited anxiogenic-like behaviors and increased $I_{\mathrm{h}}$, similar to that observed following CUS. Our results suggest that CUS, but not acute stress, leads to an increase in perisomatic $I_{\mathrm{h}}$ in dorsal CA1 neurons and that HCN channels represent a potential target for the treatment of major depressive disorder.
\end{abstract}

Molecular Psychiatry (2018) 23, 892-903; doi:10.1038/mp.2017.28; published online 18 April 2017

\section{INTRODUCTION}

The limbic-cortical areas are implicated in the pathophysiology of affective disorders. Accumulating evidence suggests that the hippocampus is an important brain region underlying the action of depression and antidepressants. ${ }^{1-6}$ On the basis of anatomical, biochemical, and functional properties, the hippocampus can be divided into dorsal and ventral regions. ${ }^{7-9}$ In patients with depression, the posterior hippocampus, analogous to the dorsal hippocampus in rodents, is significantly reduced in volume. ${ }^{1,10}$ In addition, knockdown of brain-derived neurotrophic factor (BDNF) and pharmacological manipulations of the dorsal hippocampus in rodents leads to resilient ${ }^{11,12}$ or antidepressant-like effects. ${ }^{13-17}$

Chronic stress is a significant risk factor for the development of depression. ${ }^{18}$ Prolonged stress induces dysfunction of the hypothalamus-pituitary-adrenal axis, which leads to chronic elevations of cortisol (corticosterone in rodents) in depressed patients. ${ }^{19,20}$ Chronic unpredictable stress (CUS) is a widely accepted rodent model of depression. ${ }^{21,22}$ The CUS paradigm meets three main criteria for an animal model of depression-(1) face validity (that is, depression-like symptoms similar to that observed in patients with depression), (2) construct validity (that is, CUS-induced pathophysiological changes that occur in patients with depression) and (3) predictive validity (that is, the reversal of depressive-like behaviors by clinical antidepressants). ${ }^{23}$ For example, CUS-treated rats display anhedonia (that is, a decreased preference for sucrose) and behavioral despair (increased passive activity in the forced swim test) that are core symptoms of human depression. $^{24,25}$

Hyperpolarization-activated cyclic nucleotide gated nonselective cation channels ( $\mathrm{HCN}$ channels) are highly expressed in the hippocampus. ${ }^{26}$ In the hippocampal CA1 region, HCN1 is the main pore-forming subunit of h-channels with a gradient of increasing channel density along the somatodendritic region of CA1. ${ }^{27-29}$
HCN channels are active at the resting membrane potential and contribute to the intrinsic membrane properties of CA1 neurons (for example, resting membrane potential, input resistance, action potential firing, and resonance frequency). ${ }^{30-34}$ Growing evidence suggests that HCN channels play a role in the action of antidepressant and depression. ${ }^{16,35,36}$ A reduction of functional $I_{\mathrm{h}}$, the current mediated by $\mathrm{HCN}$ channels, in the brain produces antidepressant-like effects. Mice in which either the poreforming or auxiliary subunits of h-channels were knocked out or knocked down (for example, HCN1, HCN2 and TRIP8b), ${ }^{16,36}$ exhibited antidepressant-like behaviors. Despite these studies, the physiological role of h-channels in anxiety- and depressionrelated behaviors in the hippocampus is unknown. In this study, we investigated whether there were changes in $I_{\mathrm{h}}$ and HCN1 subunit expression in the hippocampus of rats subjected to CUS. We found that CUS-induced anxiogenic- and depressive-like behaviors were correlated with increased perisomatic $I_{\mathrm{h}}$ in dorsal but not in ventral CA1 neurons. However, no such changes in $I_{\mathrm{h}}$ were observed following two different forms of acute stress. We also demonstrated that lentiviral-mediated knockdown of HCN1 protein expression in the dorsal CA1 region of CUS-treated rats produced resilient-like effects. Finally, in vivo block of SERCA calcium pumps, a manipulation known to increase intracellular calcium levels and upregulate $I_{h},{ }^{37-39}$ mimicked the behavioral and physiological phenotypes of CUS.

\section{MATERIALS AND METHODS}

Animals

Male Sprague-Dawley rats were used for this study. All behavioral and electrophysiological experiments were performed on 9-14 weeks old rats following 2-3 weeks of CUS. For viral knockdown experiments, lentivirus was stereotaxically injected into rats that were 4 weeks. This allowed for 3-4 weeks of expression period before beginning the CUS procedures.

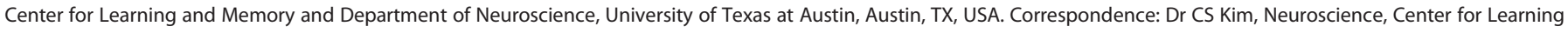
and Memory and Department of Neuroscience, University of Texas at Austin, 100 East 24th St, Austin, TX 78712-0805, USA. 
Rats were maintained on a $12 \mathrm{~h}$ light-dark schedule with ad libitum access to water and food. Rats were housed 2-3 per cage throughout the study except for CUS-treated rats subjected to stressors (for example, isolation and overcrowding; see Supplementary Table S1). The University of Texas at Austin Institutional Animal Care and Use Committee (IACUC) approved all animal procedures.

\section{Drugs}

ZD7288 was purchased from Abcam (Cambridge, MA, USA). D-APV, Gabazine, CGP 52432 and DNQX were purchased from Tocris (Bristol, UK). S-ketamine, fluoxetine, and diazepam were obtained from Sigma-Aldrich (St Louis, MO, USA).

\section{Construction of lentiviral shRNA-HCN1}

See Supplementary Information for details.

\section{Generation of lentivirus}

See Supplementary Information for details.

\section{CUS procedures}

In CUS, mild stressors were randomly administered once or twice daily at variable times of the day. Rats exposed to stress were kept in the procedure room for at least $1-2 \mathrm{~h}$ until odor from the stress procedure disappeared. The body weight of the rats was measured daily during the CUS procedure. CUS rats were subjected to the stress procedures until they were killed for electrophysiological recordings. The CUS procedure is detailed in Supplementary Table S1.

\section{Behavioral tests}

Open field test, elevated plus maze test, and forced swim test were performed as previously described. ${ }^{16}$

Open field test. Each rat was randomly assigned and placed into the center of the field. Animals in the open field arena were recorded for 6 or 11 min using a CCD camera. After each testing session, the apparatus was cleaned with $70 \% \mathrm{EtOH}$ to remove any odors deposited by previous animals. The total distance traveled (measured as line crossings) and the number of entries made into the center square was measured using a custom written program (IGOR Pro, Wavemetrics). A line crossing was defined when the body center crosses a line.

Elevated plus maze test. The animal was placed into the intersection of the four arms (center area) and the behavior was recorded for 6 min using a CCD camera. The number of open and closed arm entries was recorded along with the time spent in each arm using a custom written program (IGOR Pro, Wavemetrics). Arm entry was defined as 50\% of the body being positioned within the arm. The apparatus was cleaned with $70 \% \mathrm{EtOH}$ after each trial to remove permeated odors by previous animals.

Forced swim test. Each rat was placed in a transparent Plexiglas cylinder $\left(80 \mathrm{~cm}\right.$ tall $\times 30 \mathrm{~cm}$ in diameter) filled with water $\left(23-24^{\circ} \mathrm{C}\right)$ to a depth of $45 \mathrm{~cm}$. Water in the tank was changed after each trial. For pretest session (day 1), rats without any drug treatments were placed in the water for $15 \mathrm{~min}$. Rats were placed in the water again for a 6 min (test session) $24 \mathrm{~h}$ after the pretest session. The behaviors in the forced swim test were recorded by a video cameras positioned on the top of the water tank. Passive activity was defined as floating and making only those movements necessary to keep the nose above the water. Behaviors from the forced swim test was quantified using a custom written program which used a time sampling technique to rate the predominant behavior over a 5 -sec interval as previously described. ${ }^{40}$

\section{Sucrose preference test}

After the sequential application of a variety of mild stressors for 2-3 weeks, CUS-treated and handled control rats were habituated for 2 days to two water bottles in their home cages. The positions of bottles were counterbalanced across the left or right side of the testing cages. Following habituation, the sucrose preference of CUS-treated and control rats was tested. Volume intake of water and a $1 \%$ sucrose solution were measured during a $12 \mathrm{~h}$ dark-cycle. Sucrose preference was determined as the ratio of sucrose to total water consumption.

\section{Stereotaxic microinjection}

See Supplementary Information for details.

Dorsal CA1 cannulation and infusion. Dorsal CA1 cannulation and infusion were performed as previously described. ${ }^{13}$ Male Sprague-Dawley rats weighing $300-325 \mathrm{~g}$ were anesthetized by an intraperitoneal injection of a mixture of ketamine/xylazine $\left(90 / 10 \mathrm{mg} \mathrm{ml}^{-1} ; 0.1 \mathrm{ml}\right.$ per $100 \mathrm{~g}$ rat intraperitoneally). A stainless steel guide cannula (22 gauge; Plastics One, Roanoke, VA, USA) was bilaterally implanted $1 \mathrm{~mm}$ above the dorsal CA1 region (AP: $-3.8 \mathrm{~mm}, \mathrm{ML}: \pm 2.5 \mathrm{~mm}, \mathrm{DV}:-1.4 \mathrm{~mm}$ ) and secured with screws in the skull and dental acrylic cement. After stereotaxic implantation, a dummy cannula (Plastics One) was inserted into each guide cannula to maintain patency. Rats received food soaked with Baytril $(0.3 \mathrm{ml}$; Bayer, Shawnee Mission, KS, USA), an antibiotic as prophylaxis against infection as well as triple antibiotic ointment (Select Medical Product) applied around the surgery areas. After a minimum 5-day recovery period, rats were divided into two groups: control rats were infused with vehicle $(0.1 \%$ DMSO in aCSF; $n=11)$ and experimental rats were infused with $1 \mu \mathrm{M}$ thapsigargin $(n=14)$. All infusions ( $1 \mu$ l per side for $5 \mathrm{~min}$ ) were performed in their home cages $40 \mathrm{~min}$ before the open field test. After completion of the infusion, the injector was left in place for 5 additional min before withdrawal. After a 10-min open field test, acute dorsal hippocampal slices were immediately prepared for whole-cell current-clamp recordings. All experiments were performed under blind conditions.

\section{Slice preparation}

Rats were anesthetized with a lethal dose of a ketamine/xylazine mixture (90/10 $\mathrm{mg} \mathrm{ml}^{-1} ; 0.2-0.3 \mathrm{ml}$ per $100 \mathrm{~g}$ rat intraperitoneally) and transcardially perfused with ice-cold artificial cerebral spinal fluid (aCSF) composed of (in mm): $2.5 \mathrm{KCl}, 1.25 \mathrm{NaH}_{2} \mathrm{PO}_{4}, 25 \mathrm{NaHCO}_{3}, 0.5 \mathrm{CaCl}_{2}, 7 \mathrm{MgCl}_{2}, 7$ dextrose, 210 sucrose, 1.3 ascorbic acid and 3 sodium pyruvate, bubbled with $95 \% \mathrm{O}_{2}-5 \% \mathrm{CO}_{2}$. The brain was removed and hemisected along the longitudinal fissure. Dorsal and ventral hippocampal slices were prepared as previously described (Supplementary Figure S1). ${ }^{41} 300 \mu \mathrm{m}$ thick hippocampal slices were made in ice-cold aCSF using a vibrating microtome (Microslicer DTK-Zero1, DSK, Kyoto, Japan). Half the slices were placed in a holding chamber containing (in $\mathrm{mM}$ ) $125 \mathrm{NaCl}, 2.5 \mathrm{KCl}, 1.25$ $\mathrm{NaH}_{2} \mathrm{PO}_{4}, 25 \mathrm{NaHCO}_{3}, 2 \mathrm{CaCl}_{2}, 2 \mathrm{MgCl}_{2}, 12.5$ dextrose, 1.3 ascorbic acid, and 3 sodium pyruvate, bubbled with $95 \% \mathrm{O}_{2}-5 \% \mathrm{CO}_{2}$ at $35^{\circ} \mathrm{C}$ for $30 \mathrm{~min}$ and then incubated for at least $45 \mathrm{~min}$ at room temperature before used for electrophysiology. The remaining slices were fixed overnight in $4 \%$ paraformaldehyde and then placed into $30 \%$ sucrose in PBS for immunohistochemistry.

Somatic and dendritic whole-cell current-clamp recordings

Whole-cell current-clamp recordings were performed as previously described. ${ }^{16,41}$ Briefly, hippocampal slices were submerged in a recording chamber continuously perfused with aCSF containing (in mM) $125 \mathrm{NaCl}$, $3 \mathrm{KCl}, 1.25 \mathrm{NaH}_{2} \mathrm{PO}_{4}, 25 \mathrm{NaHCO}_{3}, 2 \mathrm{CaCl}_{2}, 1 \mathrm{MgCl}_{2}$ and 12.5 dextrose, bubbled with $95 \% \mathrm{O}_{2}-5 \% \mathrm{CO}_{2}$ at a rate of $1 \mathrm{ml} \mathrm{min}^{-1}$ and $32-34{ }^{\circ} \mathrm{C}$. Synaptic blockers (D-APV $25 \mu \mathrm{m}$, Gabazine $2 \mu \mathrm{m}$, CGP $558455 \mu \mathrm{m}$ and DNQX $20 \mu \mathrm{M})$ were included to block synaptic transmission. CA1 pyramidal neurons were visually identified using a microscope (Zeiss Axioskop, Thornwood, NY, USA) fitted with differential interference contrast optics. ${ }^{42}$ Patch pipettes for somatic (4-7 M $\Omega$ ) and dendritic recordings (7-8 M $\Omega$ ) were prepared with capillary glass (external diameter $1.65 \mathrm{~mm}$ and internal diameter $1.1 \mathrm{~mm}$, World Precision Instruments) using a Flaming/Brown micropipette puller and filled with an internal solution containing (in $\mathrm{mm}$ ) 120 K-gluconate, $20 \mathrm{KCl}, 10 \mathrm{HEPES}, 4 \mathrm{NaCl}, 7 \mathrm{~K} 2$-phosphocreatine, $4 \mathrm{Mg}$-ATP, $0.3 \mathrm{Na}-\mathrm{GTP}$ (pH 7.3 with $\mathrm{KOH}$ ). Neurobiotin $(0.1-0.2 \%$, Vector Labs) was included for subsequent histological processing. Whole-cell current-clamp recordings were performed using a Dagan BVC-700A amplifier (Dagan, Minneapolis, MN, USA) and custom acquisition software written in IGOR Pro. Electrical signals were filtered at $5-10 \mathrm{kHz}$, sampled at $20-40 \mathrm{kHz}$, and digitized by an ITC-18 interface (Instructech, Port Washington, NY, USA). Series resistance was monitored during each experiment and experiments were discarded if the series resistance exceeded $25 \mathrm{M} \Omega$ for somatic recordings and $20 \mathrm{M} \Omega$ for dendritic recordings. The 'natural' resting membrane potential was the potential of the soma or dendrite in the absence of any injected current. Each 


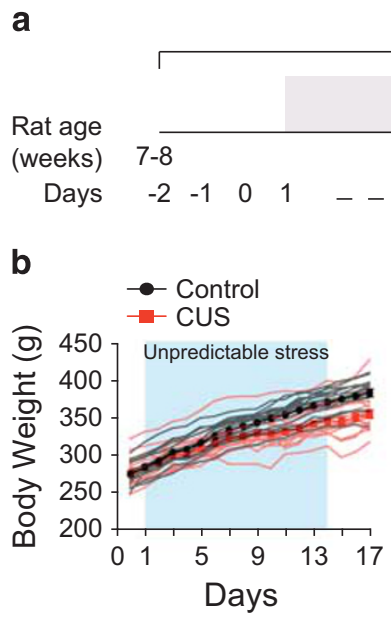

g
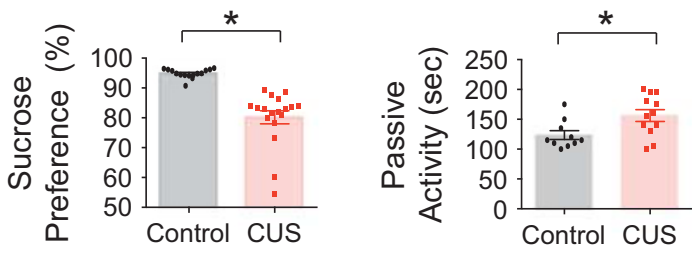

Body weight

\begin{tabular}{|c|c|c|c|}
\hline CUS & $\begin{array}{l}\text { Sucrose Preference } \\
\text { (Single housing) }\end{array}$ & Behavioral tests & Electrophysiology \\
\hline
\end{tabular}

h
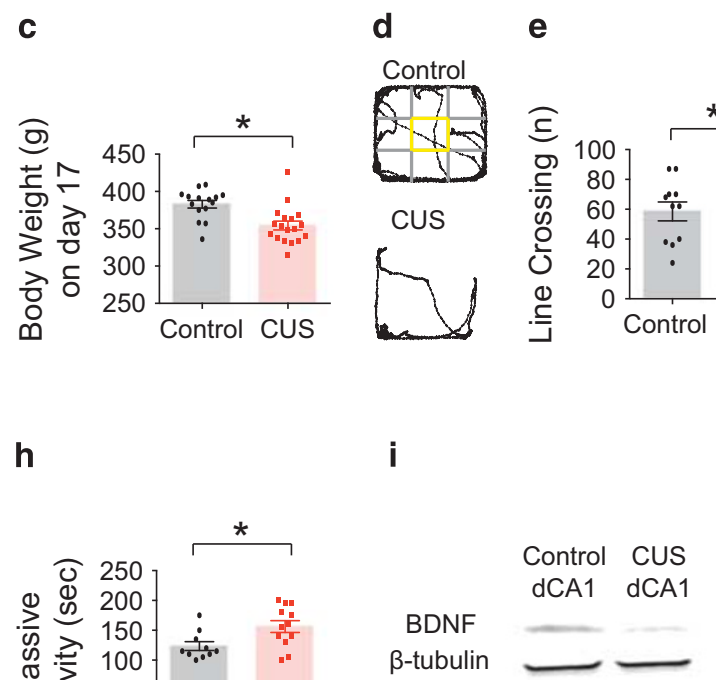

d

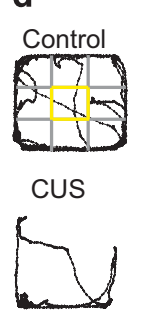

e

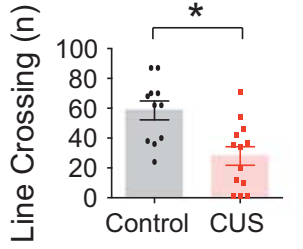

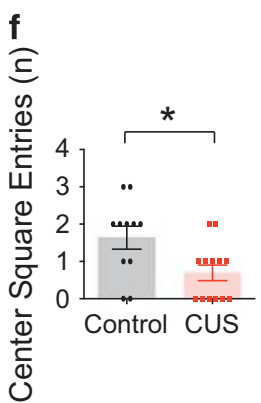

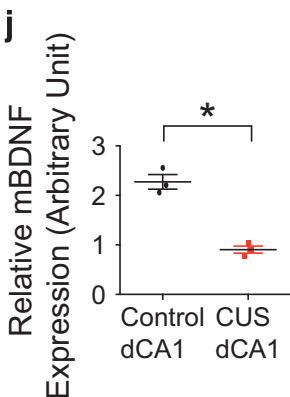

k

Dorsal Hippocampus
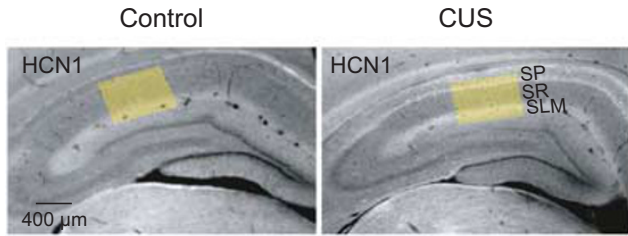

m

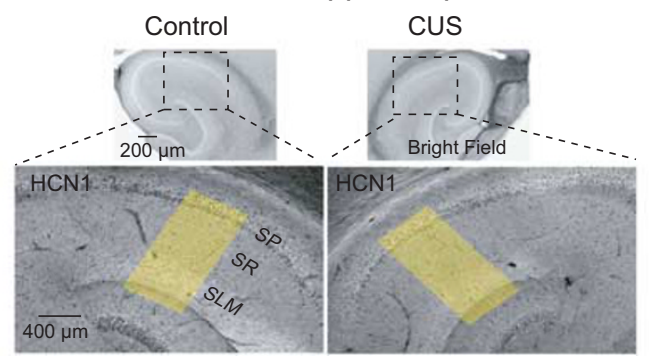

n HCN1 Protein Expression

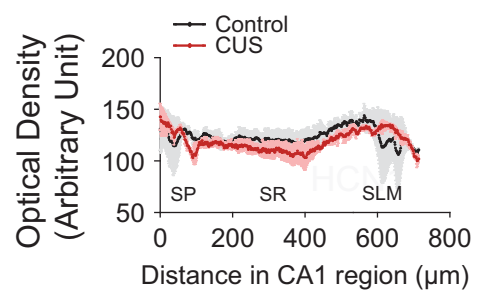

Figure 1. CUS increased HCN1 protein expression in dorsal but not in ventral CA1 region. (a) Timeline of chronic unpredictable stress, behavioral tests, and electrophysiology. (b) Rats subjected to chronic unpredictable stress gained less body weight than those from control rats. (c) On day 17, CUS-treated rats showed significantly decreased body weight compared to those from control rats. (d) Representative video tracking images during the last $5 \mathrm{~min}$ of open field test of age-matched individual rats-control vs CUS-treated rats. CUS-treated rats showed decreases in line crossing (e) and center square entries (f). (g and $\mathbf{h}$ ) CUS-treated rats displayed decreased sucrose preference (i.e., anhedonic-like effect) and increased passive activity time (i.e., behavioral despair-like effect). (i) Representative bands from western blotting of control and CUS groups. (j) Mature BDNF (mBDNF) protein expression was normalized to $\beta$-tubulin. mBDNF protein expression was significantly decreased in CUS group compared to control group. (k and $\mathbf{m}$ ) Representative dorsal and ventral hippocampal slices immunolabeled with antibody against HCN1. Yellow boxes depict the region of the slice used for quantification of the optical density. (I and $\mathbf{~ m ) ~ Q u a n t i f i c a t i o n ~ o f ~ H C N 1 ~ p r o t e i n ~ e x p r e s s i o n ~ f r o m ~ p e r i s o m a t i c ~ t o ~ d i s t a l ~ d e n d r i t i c ~ r e g i o n ~ o f ~ C A 1 ~ i n ~ d o r s a l ~ a n d ~ v e n t r a l ~ h i p p o c a m p u s . ~}$ Data are expressed as mean \pm s.e.m. with significance indicated by ${ }^{*} P<0.05$, compared with control group. BDNF, brain-derived neurotrophic factor; CUS, chronic unpredictable stress. 
measurement was also made at a fixed or common membrane potential of $-73 \mathrm{mV}$. All membrane potentials were corrected for liquid junction potentials $(-8 \mathrm{mV})$

\section{Cell-attached patch clamp recordings}

For cell-attached recordings, pipettes contained the following (in $\mathrm{mm}$ ): 120 $\mathrm{KCl}, 10 \mathrm{HEPES}, 2.0 \mathrm{CaCl}_{2}, 1.0 \mathrm{MgCl}_{2}, 20 \mathrm{TEA}-\mathrm{Cl}, 54-\mathrm{AP}, 1 \mathrm{BaCl}_{2}, \mathrm{pH} 7.3$ with $\mathrm{KOH}$. Membrane currents were recorded using an Axopatch 200B amplifier (Molecular Devices, Sunnyvale, CA, USA), sampled at $10 \mathrm{kHz}$, analog filtered at $2 \mathrm{kHz}$, and digitized by an ITC-18 interface connected to a computer running Axograph $\mathrm{X}$. Maximum $\mathrm{I}_{\mathrm{h}}$ was measured by using hyperpolarizing voltage commands to $-140 \mathrm{mV}$ from a holding potential of $-30 \mathrm{mV}$. Linear leakage and capacitive currents were digitally subtracted by scaling traces at smaller command voltages in which no voltage-dependent current was activated. All experiments were performed under blind conditions.

Neuronal reconstructions

See Supplementary Information for details.
Immunohistochemistry and western blotting

See Supplementary Information for details.

Data analysis. Input resistance was measured by the slope of the linear fit of the V-I plot between +10 and -150 pA current injections. The slow time constant was calculated from a double-exponential fit of the averaged voltage decay resulting from 50 or 100 trials of identical 1-ms, 200 or $400-p A$ current injections. Resonance frequency was measured as the frequency of the peak impedance using a sinusoidal current injection of constant amplitude and linearly spanning $0-15 \mathrm{~Hz}$ in $15 \mathrm{~s}$. The total length of CA1 was measured along the CA1 neuron layer between the CA2/CA1 border $\left(\mathrm{X}_{1}\right)$ and the CA1/subicular border $\left(\mathrm{X}_{2}\right) .{ }^{43}$ Data were not excluded from analysis in this study.

Statistical analysis. Statistical comparisons were performed using analysis of variance (one factor or two factor) followed by Bonferrori post hoc test, paired $t$-test (Wilcoxon signed-rank test), or unpaired $t$-test (Mann-Whitney U-test) with GraphPad software (La Jolla, CA, USA). ${ }^{*} P<0.05$ was considered as statistically significant.

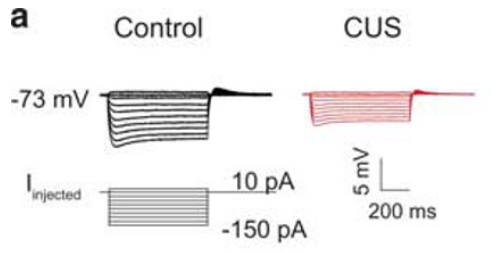

b

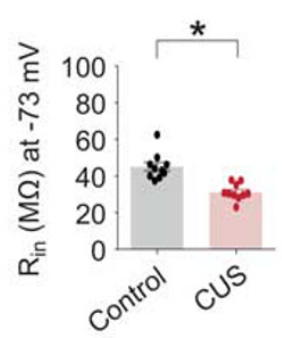

h

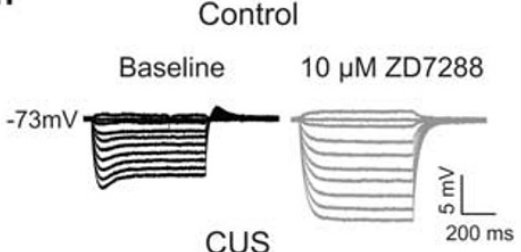

CUS
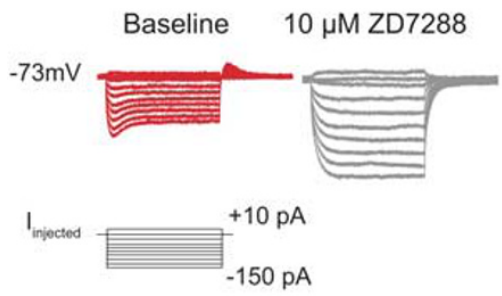

c Control

cus

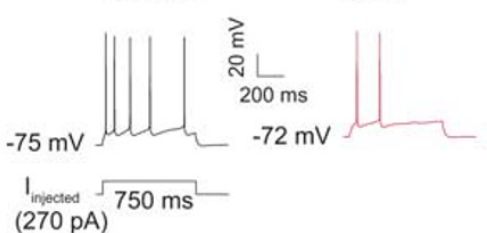

e

Control

cus

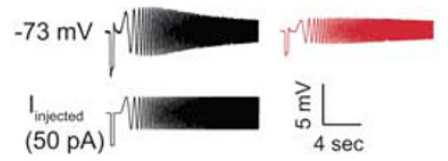

f

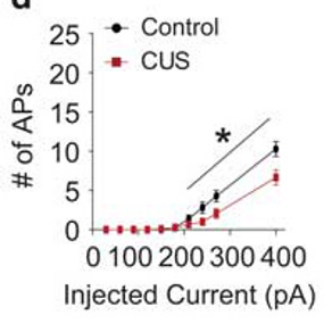

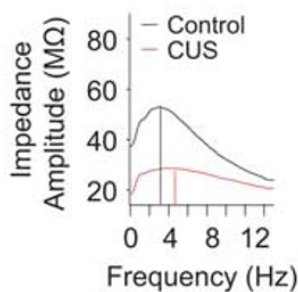

g

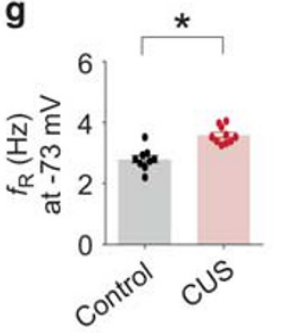

i

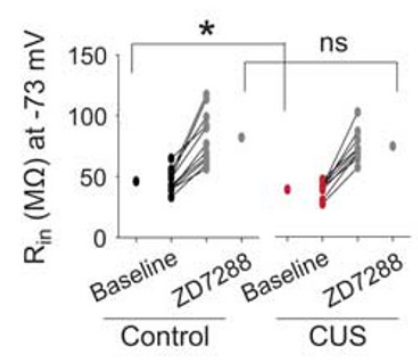

j

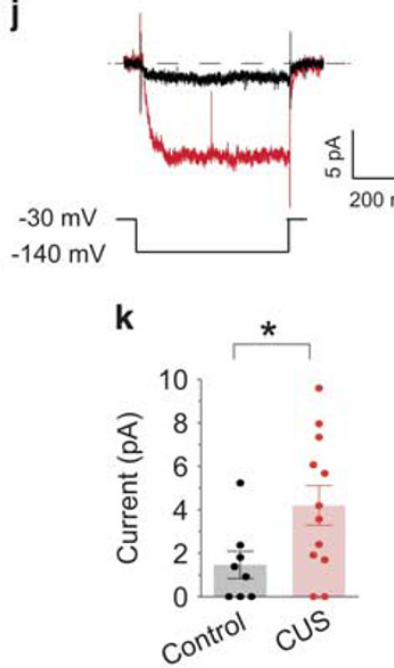

Figure 2. Upregulation of $I_{\mathrm{h}}$-related electrophysiological measurements in dorsal CA1 neurons following CUS (a) Representative voltage responses with step current injections at a common membrane potential $(-73 \mathrm{mV} ; 750 \mathrm{~ms})$. (b) Input resistance ( $\left.R_{\text {in }}\right)$ at $-73 \mathrm{mV}$ was significantly reduced in dorsal CA1 neurons following CUS. (c) Representative voltage responses with depolarizing current step (270 pA; $750 \mathrm{~ms}$ ) at resting membrane potential. (d) CUS led to a decrease in action potential firing in dorsal CA1 neurons compared to control group. (e) Representative voltage traces and current injections at $-73 \mathrm{mV}$. (f) The profile of impedance amplitude for voltage traces in e. Vertical lines indicate the resonance frequencies. (g) Resonance frequency was significantly increased in dorsal CA1 neurons following CUS. (h) Representative voltage responses with step current injections at common membrane potential ( $-73 \mathrm{mV}$; $750 \mathrm{~ms})$. (i) Bath application of $10 \mu \mathrm{m}$ ZD7288 showed similar changes in $R_{\text {in }}$ in dorsal CA 1 neurons from control and CUS groups. (j) $/ \mathrm{h}$ was measured by stepping from a holding potential of $-30 \mathrm{mV}$ to $-140 \mathrm{mV}$ for $500 \mathrm{~ms}$. (k) $/ \mathrm{h}$ was significantly increased in CUS group compared to control group. Data are expressed as mean \pm s.e.m. with significance indicated by ${ }^{*} P<0.05$, compared with control group. CUS, chronic unpredictable stress. 
a

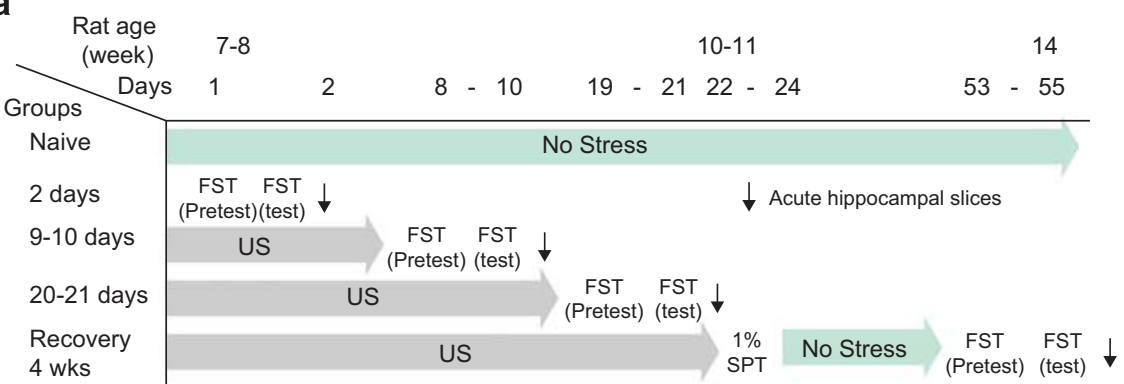

C

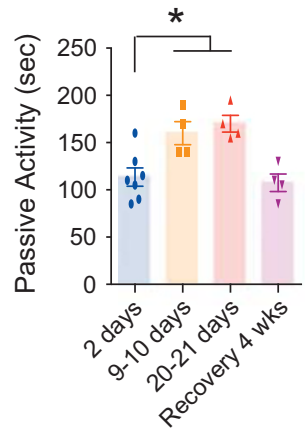

d

Before a 4-week recovery period

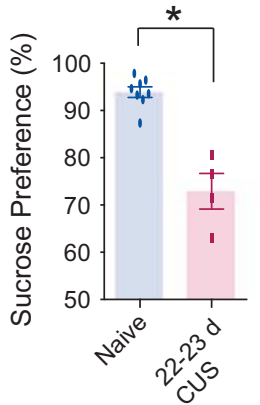

k
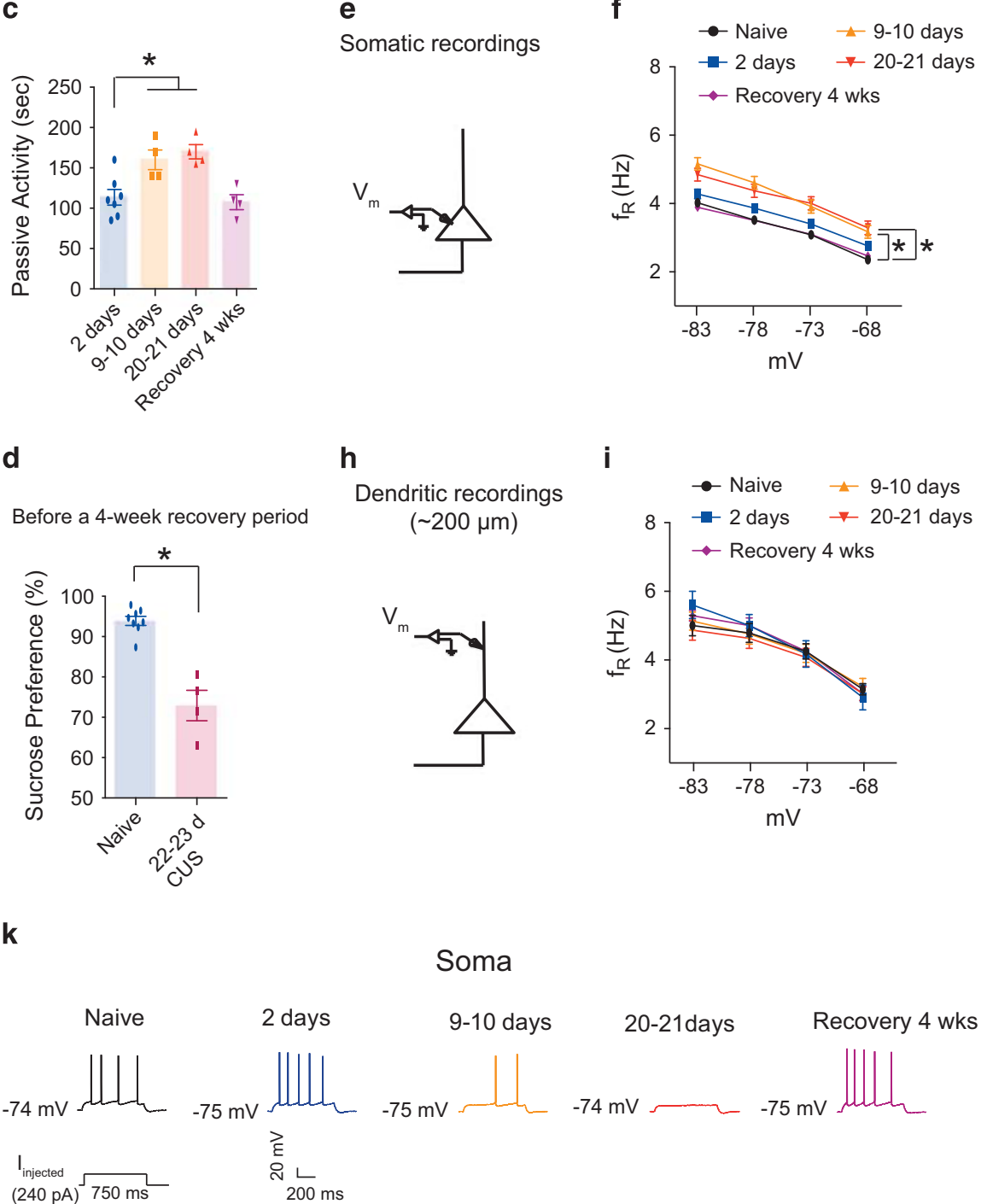

i

Dendritic recordings

$(\sim 200 \mu \mathrm{m})$
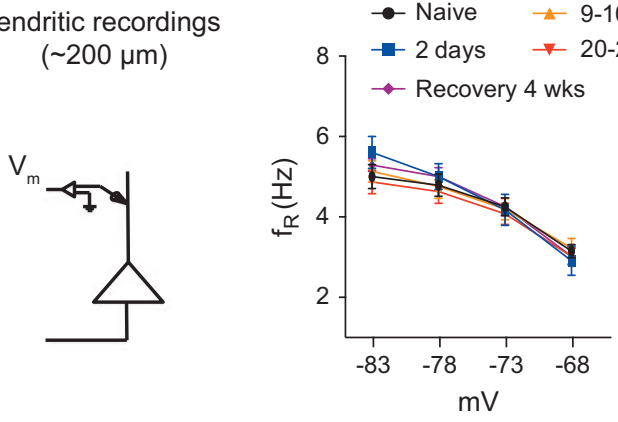

b

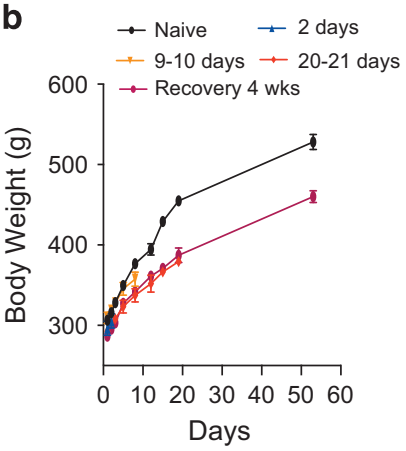

g

j
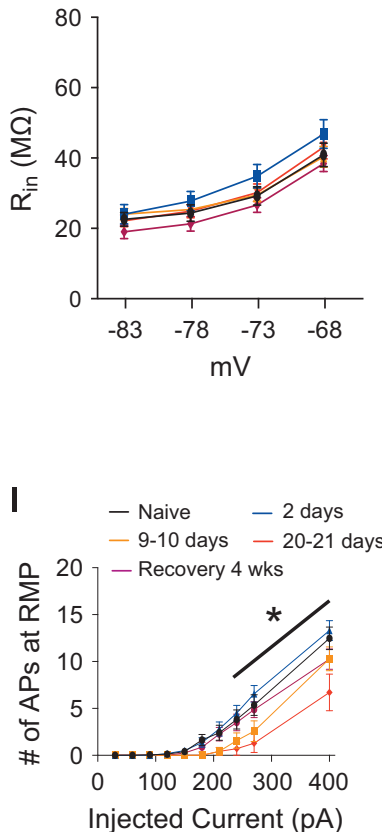

Figure 3. Upregulation of perisomatic but not dendritic $I_{\mathrm{h}}$ in dorsal CA1 neurons correlated with the time course of CUS. (a) Timeline of the experimental procedure. (b) Body weight changes during the time course of CUS. (c) Passive activity time gradually increased with increasing duration of CUS. Rats receiving a 4-week recovery period showed no persistent behavioral despair. (d) Rats subjected to 22-23 days CUS displayed a decreased sucrose preference (i.e., anhedonic-like effect) compared to naive rats. (e) Schematic of the somato-apical trunk depicting the somatic recordings. (f) $f_{\mathrm{R}}$ was significantly increased from 9-10 days CUS to 20-21 days CUS compared to naive group at each membrane potential. This CUS-induced an increase in $f_{\mathrm{R}}$ did not occur after a 4-week recovery period. (g) $R_{\text {in }}$ was significantly decreased after 20-21 days CUS compared to naive group at each membrane potential except at $-83 \mathrm{mV}$. This CUS-induced a reduction in $\mathrm{R}_{\text {in }}$ did not occur after a 4-week recovery period compared to naive group. (h) Schematic of the somato-apical trunk depicting the dendritic recordings. (I and J) $f_{R}$ and $R_{\text {in }}$ were not significantly altered during the time course of CUS or after a 4-week recovery time. (k) Representative voltage responses with depolarizing current step $(240 \mathrm{pA} ; 750 \mathrm{~ms})$ at RMP. (I) Action potential firing was gradually decreased with increasing duration of CUS. This CUS-induced decrease in action potential firing did not occur after a 4-week recovery period. Data are expressed as mean \pm s.e.m. with significance indicated by ${ }^{*} P<0.05$, compared with naive group or 20-21 days CUS group. CUS, chronic unpredictable stress; FST, forced swim test; RMP, resting membrane potential; SPT, sucrose preference test; US, unpredictable stress. 


\section{RESULTS}

HCN1 protein and $I_{\mathrm{h}}$ are increased in dorsal, but not in ventral CA1 region/neurons following chronic unpredictable stress

Rats were subjected to CUS for 2-3 weeks (Figure 1a and Supplementary Table S1), and their body weight was measured daily (Figure 1b). CUS-treated rats showed a significantly lower body weight on day 17 compared to those from control rats (Figure 1c and Supplementary Table S2). CUS-treated rats showed reduced locomotor activity and center square entries in the open field test (Figures $1 \mathrm{~d}, \mathrm{f}$ and Supplementary Table S2). Consistent with depression in humans, ${ }^{24}$ CUS led to anhedonia, observed as a decreased sucrose preference (Figure $1 \mathrm{~g}$ and Supplementary Table S2) and behavioral despair, observed as increased passive activity (Figure $1 \mathrm{~h}$ and Supplementary Table S2), compared to control rats. BDNF is considered a biological marker for depression. ${ }^{5,44}$ We therefore measured BDNF expression in control and CUS-treated rats. Indeed, BDNF protein expression in the dorsal CA1 region was significantly reduced following CUS (Figures $1 \mathrm{i}$ and $\mathrm{j}$ ). The results of both the behavioral and biochemical testing confirm the validity of the CUS model in our hands. After behavioral testing, we performed immunohistochemical staining for the HCN1 subunit of h-channels in the dorsal and ventral hippocampus. Interestingly, perisomatic expression of HCN1 protein in the dorsal CA1 region was significantly increased, with no difference in dendritic expression (Figures $1 \mathrm{k}$ and I). There was no difference in $\mathrm{HCN} 1$ protein expression in the $\mathrm{CA} 1$ region of the ventral hippocampus (Figures $1 \mathrm{~m}$ and $\mathrm{n}$ ).

To further explore this finding, we compared the electrophysiological properties of dorsal and ventral CA1 neurons between control and CUS-treated rats. Following behavioral testing, acute hippocampal slices were prepared and $I_{\mathrm{h}}$-sensitive electrophysiological measurements were determined using somatic whole-cell current-clamp recordings. ${ }^{16,34}$ Input resistance $\left(R_{\text {in }}\right)$ at a common membrane potential $(-73 \mathrm{mV})$ was significantly lower in dorsal CA1 neurons from CUS-treated rats compared to those from control rats (Figures $2 \mathrm{a}, \mathrm{b}$ and Supplementary Table S3). The number of action potentials at the resting membrane potential (RMP) was significantly lower in dorsal CA1 neurons from CUStreated rats compared to those from control rats (Figures $2 c$, d and Supplementary Table S3), suggesting a decrease in neuronal excitability. Additionally, resonance frequency $\left(f_{\mathrm{R}}\right)$ at $-73 \mathrm{mV}$ was significantly increased in dorsal CA1 neurons from CUS-treated rats compared to those from control rats (Figures $2 \mathrm{e}-\mathrm{g}$, and Supplementary Table S3). There were no significant differences in $\mathrm{R}_{\mathrm{in}}, f_{\mathrm{R}}$, or the number of action potentials measured in ventral CA1 neurons between CUS-treated and control rats (Supplementary Figure S2 and Supplementary Table S3). In agreement with these results, Lentiviral-mediated reduction of $\mathrm{HCN} 1$ protein expression in the ventral CA1 region had no effect on anxiolytic(Supplementary Figures S3E and $\mathrm{S} 2 \mathrm{H}$ ) or antidepressant-like (Supplementary Figure S3I) behaviors. The lentiviral-mediated knockdown of HCN1 was confirmed by physiological measurements from ventral $C A 1$ neurons after behavioral testing (Supplementary Figures S3J-S3P).

The magnitudes of $R_{\text {in }}$ and $f_{R}$ are dependent upon the longitudinal location of neurons within the CA1 region and on neuronal morphology. ${ }^{45-47}$ CA1 neurons were filled with neurobiotin during electrophysiological recording and fixed for post-hoc morphological analysis. The total length of CA1 and the position of recorded CA1 neurons were not significantly different between control and CUS groups in dorsal (Supplementary Figures S4AS4D) or ventral (Supplementary Figures S4E-S4H) hippocampus. Morphological three-dimensional reconstruction of CA1 neurons revealed that dendrite length and number of branches for basal (Supplementary Figures S5A and S5B) and apical (Supplementary Figures S5A and $\mathrm{S} 5 \mathrm{C}$ ) dendrites were not significantly different between control and CUS groups in either the dorsal or ventral hippocampus. In addition, Sholl analysis showed no significant differences in overall dendritic branching patterns between control and CUS groups in dorsal (Supplementary Figure S5D) or ventral (Supplementary Figure S5E) hippocampus.

The results thus far suggest that CUS increases perisomatic $I_{\mathrm{h}}$ in dorsal, but not ventral CA1 neurons. As a further test of this hypothesis we compared the effects of $10 \mu \mathrm{M}$ ZD7288, a blocker of HCN channels, on dorsal CA1 neurons from control and CUS groups. ZD7288 was applied for 4 to 5 min before switching back to the normal aCSF. ${ }^{41}$ As expected, brief bath application of ZD7288 significantly hyperpolarized $V_{m}$ and increased $R_{\text {in }}$ in control and CUS groups (Supplementary Table S4). After block of $I_{\mathrm{h}}$ by ZD7288, there were no significant differences in $R_{\text {in }}$ at either the RMP or $-73 \mathrm{mV}$ between control and CUS groups (Supplementary Table S4). $R_{\text {in }}$ at $-73 \mathrm{mV}$ was significantly increased in the presence of ZD7288 in control and CUS groups (Figures $2 \mathrm{~h}$, i and Supplementary Table S4). Finally, we used cellattached patch clamp recordings to directly measure $I_{\mathrm{h}}$ at the soma of dorsal CA1 neurons from control and CUS-treated rats (Figures $2 \mathrm{j}$ and $\mathrm{k}$ ). Patches from CUS-treated rats had significantly more $I_{\mathrm{h}}$ compared to patches from control rats (Figure $2 \mathrm{k}$ ). These results suggest that CUS lowers CA1 pyramidal neuron excitability in part by upregulation of perisomatic HCN1 subunit expression and $I_{\mathrm{h}}$.

Upregulation of perisomatic, but not dendritic $I_{h}$, was correlated with the time course of CUS

The forced swim test (FST) was used to determine passive activity prior to electrophysiological recordings. As the FST is an acute stressor in and of itself, we examined whether exposure to an acute stress also produced changes in $I_{\mathrm{h}}$-related measurements. Current-clamp recordings were made from dorsal hippocampal slices that were prepared immediately after rats were subjected to a 30-min restraint stress. Unlike after CUS, 30-min of acute stress had no effects on subthreshold membrane properties (for example, RMP, $\mathrm{R}_{\mathrm{in}}, f_{\mathrm{R}}$ and time constant; Supplementary Figure S6), suggesting that acute stress does not affect $I_{\mathrm{h}}$.

In the experiments described in Figures 1 and 2, rats were subjected to CUS for 2-3 weeks prior to behavioral testing and electrophysiological recordings. To determine the time course of the changes in functional $I_{\mathrm{h}}$, we performed electrophysiological recordings following different times of CUS exposure. Rats were divided into 5 groups-(1) naive (no stress), (2) 2-day stress (for example, 2-day forced swim test), (3) 9- to 10-day unpredictable stress (US), (4) 20- to 21-day US and (5) recovery for 4 weeks after last US (Figure 3a). Passive activity, sucrose preference and body weight were measured during the time course of CUS exposure. CUS-treated rats gained weight at a reduced rate compared to control rats during the CUS period, but there was no significant difference in the rate of weight gain during the recovery period (Figure 3b). Passive activity was higher after 9-10 days of CUS compared to 2-day FST (Figure 3c), but there was no further increase in passive activity after 20-21 days of CUS (Figure 3c). There was, however, a positive relationship between passive activity and sucrose preference in rats exposed to CUS (Supplementary Figure S7A). We therefore used the sucrose preference test to ascertain the onset of depressive symptoms before applying a 4-week no stress period for the recovery group. We observed that rats subjected to 22-23 days of CUS displayed a decreased sucrose preference as compared to naive group (Figure 3d). This same group was then given a 4-week recovery period followed by a sucrose preference test. After the recovery period, passive activity was not significantly different between recovery group and 2 -day FST group (Figure $3 \mathrm{c}$ ), suggesting that continued exposure to CUS is required for maintaining depressive symptoms. Because we observed depressive phenotypes during the time course of CUS, we next examined whether these changes 
in behavior were correlated with increased $I_{\mathrm{h}}$. Dorsal hippocampal slices were prepared immediately after a 6-min FST at each of the time points listed above (Figure 3a). There were no differences in either the somatic or dendritic RMP between naive and CUS groups at any time point (Supplementary Table S5). In somatic recordings, $R_{\text {in }}$ at $-73 \mathrm{mV}$ was significantly lower following
9-10 days of CUS compared to the naive group. However, it was not further decreased in 20-21 days CUS group compared to 9-10 days CUS group (Supplementary Figures S8A, S8B and Supplementary Table S5). Given that activation of $I_{h}$ is voltagedependent, we measured $\mathrm{R}_{\text {in }}$ and $f_{\mathrm{R}}$ at different membrane potentials (ranging from $-68 \mathrm{mV}$ to $-83 \mathrm{mV}$ ) in dorsal CA1

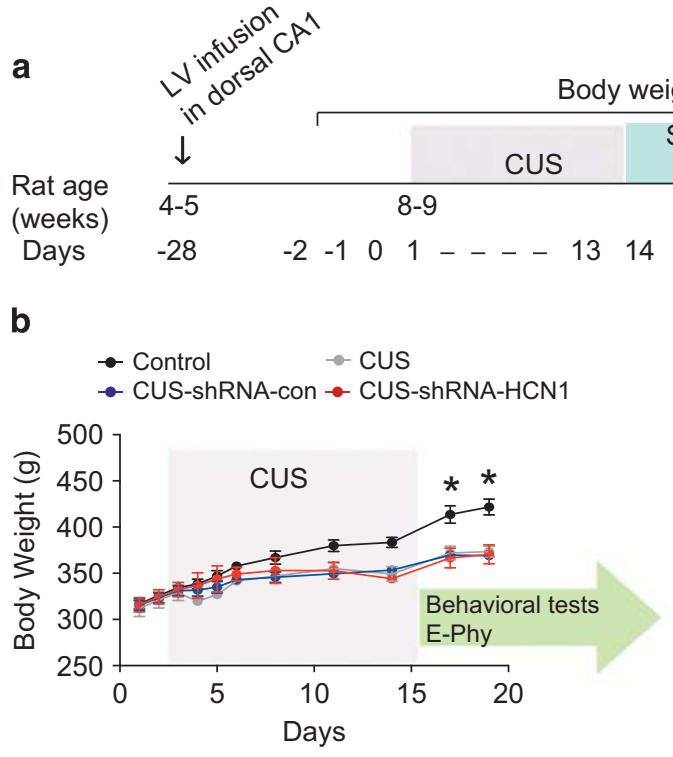

e

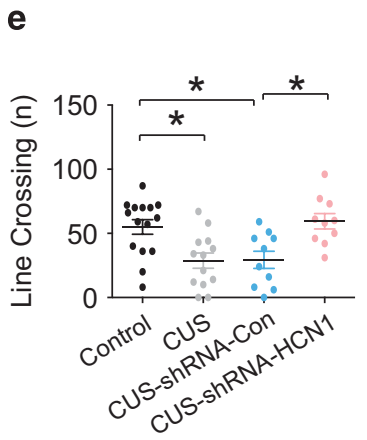

i

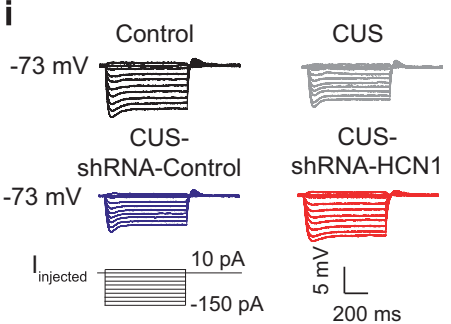

j

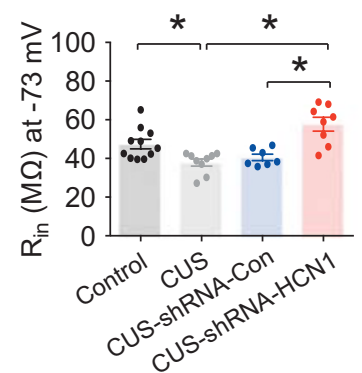

f

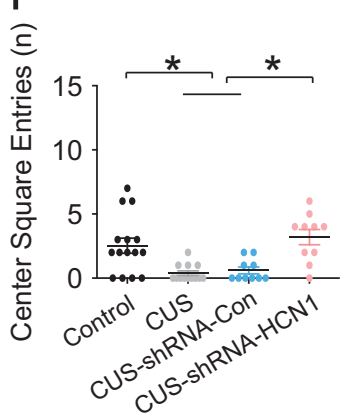

k

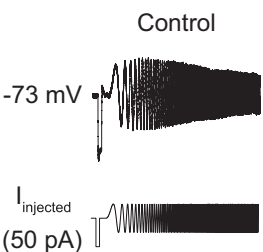

g

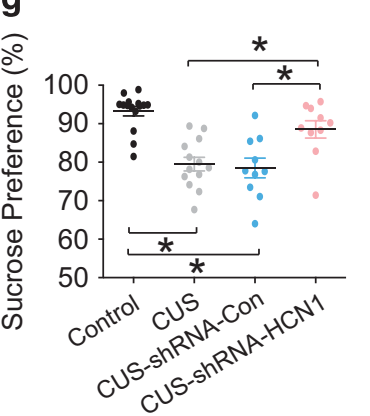

h

CUS-shRNA-control

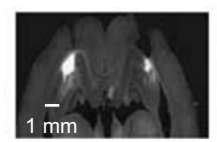

CUS-shRNA-HCN1

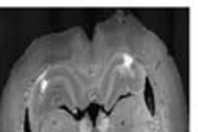

d

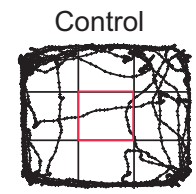

CUS-shRNA-control

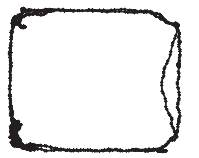

Electrophysiology

11-12 14-15

$2021 \ldots 46$


neurons (Figures 3f, g and Supplementary Figures S9). As expected, somatic $I_{\mathrm{h}}$-dependent measures gradually changed over the time course of CUS (Figures $3 f$ and g). Interestingly, $f_{\mathrm{R}}$ in 9-10 days CUS group was not significantly different from that in 20-21 days CUS group (Figure $3 \mathrm{f}$ ), consistent with the behavioral results (Figure $3 \mathrm{C}$ ). In addition, these CUS-induced changes in $I_{\mathrm{h}}$ were no longer apparent in the recovery group (Figures $3 \mathrm{f}$ and $\mathrm{g}$ ). Given that intrinsic neuronal excitability was significantly decreased after 2 to 3 weeks of CUS (Figures $2 c$ and d), we also examined whether these changes in excitability occurred during the time course of CUS. Indeed, action potential firing gradually decreased with increasing duration of CUS and was significantly different in the 20-21 days CUS group compared to either the naive or the 2-day FST group (Figures 3k, I and Supplementary Table S5). Action potential firing was not significantly different between the naive, 2-day FST, and recovery groups (Figures 3k, I and Supplementary Table S5). Because our immunohistochemical results did not show a difference in HCN1 staining in the dendritic region of CA1 between control and CUS groups (Figure 1), the changes in $I_{\mathrm{h}}$ following CUS appear to be restricted to the perisomatic region. To test this, we used whole-cell dendritic recording ( $200 \mu \mathrm{m}$ from the soma) to test whether there are functional changes in dendritic $I_{h}$ following CUS. At $-73 \mathrm{mV}$, dendritic $R_{\text {in }}$ was not significantly altered during the time course of CUS (Supplementary Figures S8C, S8D and Supplementary Table S5). In addition, dendritic measures of $I_{h}$ at different membrane potentials were not altered during the time course of CUS (Figures 3i, $\mathbf{j}$ and Supplementary Table S5). These results suggest that upregulation of perisomatic, but not dendritic $I_{\mathrm{h}}$ occurred in dorsal CA1 neurons during the time course of CUS.

\section{A reduction of $\mathrm{HCN} 1$ protein produced resiliency to CUS}

We have thus far demonstrated that perisomatic HCN1 expression and functional $I_{\mathrm{h}}$ was higher in the dorsal CA1 neurons following CUS. We previously showed that lentiviral-mediated knockdown of $\mathrm{HCN} 1$ protein and functional $I_{\mathrm{h}}$ in dorsal CA1 neurons produces an antidepressant phenotype in normal rats. ${ }^{16}$ Therefore, we next asked whether in vivo reduction of HCN1 protein expression and reduction of $I_{\mathrm{h}}$ confers resiliency to CUS. Lentivirus expressing shRNA-control or shRNA-HCN1 was injected into the dorsal CA1 region 2-3 weeks before exposure to CUS (Figure 4a). Following CUS, there was no significant difference in locomotor activity or the number of center square entries between CUS-no injection and CUS-shRNA-control injection (Figures $4 \mathrm{~d}-\mathrm{f}$ and Supplementary Table S6). More importantly, the performance of rats that received shRNA-HCN1 was not significantly different from control rats that were not subjected to CUS (Figures $4 \mathrm{~d}-\mathrm{f}$ and Supplementary Table S6). In agreement, rats that received
shRNA-HCN1 did not show a CUS-dependent decrease in sucrose preference (Figure $4 \mathrm{~g}$ and Supplementary Table S6) or an increase in passive activity in the forced swim test (Figure $4 \mathrm{~h}$ and Supplementary Table S6). Following behavioral testing, electrophysiological recordings were made from dorsal CA1 neurons. CUS-shRNA-HCN1-infected CA1 neurons had a hyperpolarized membrane potential compared to either CUS-no injection or CUSshRNA-control-infected dorsal CA1 neurons (Supplementary Table S6). Consistent with a loss of functional $I_{h}$ in the CUSshRNA-HCN1 group, $R_{\text {in }}$ was significantly higher (Figures $4 i, j$ and Supplementary Table $\mathrm{S} 6$ ) and $f_{\mathrm{R}}$ significantly lower (Figures $4 \mathrm{k}-\mathrm{m}$ and Supplementary Table S6) at $-73 \mathrm{mV}$ in dorsal CA1 neurons from CUS-shRNA-control treated rats. These changes were not present in dorsal CA1 neurons from CUS-shRNA-HCN1 treated rats. The results suggest that a reduction in HCN1 protein expression in the dorsal CA1 region is sufficient to provide resiliency to CUS.

Inhibition of SERCA pump in dorsal CA1 region/neurons leads to anxiogenic-like behavior and upregulation of $I_{\mathrm{h}}$

Chronic stress is associated with an increase in L-type calcium channel activation and hypersecretion of glucocorticoid hormones. ${ }^{48,49}$ Interestingly, an elevated basal intracellular calcium concentration in platelets and lymphocytes occurs in patients with depression. ${ }^{50,51}$ We previously reported that in vitro block of the sarcoplasmic/endoplasmic reticulum $\mathrm{Ca}^{2+}$-ATPase (SERCA) in CA1 neurons, which regulates intracellular $\mathrm{Ca}^{2+}$ levels, as well as increases in intracellular $\mathrm{IP}_{3}$, results in perisomatic increases in $I_{\mathrm{h}}{ }^{37,39,52}$ Given that CUS led to increased perisomatic $I_{\mathrm{h}}$ in dorsal CA 1 neurons, we hypothesized that in vivo block of the SERCA pumps in the dorsal CA1 region would produce the same depressive-like behavioral phenotypes observed after CUS. To test this hypothesis, we infused $1 \mu \mathrm{m}$ thapsigargin (TG), an irreversible inhibitor of the SERCA pump, into the dorsal CA1 region via an implanted cannula. Previous studies showed that $1 \mu \mathrm{m}$ TG effectively leads to depletion of intracellular calcium stores and increases intracellular cytosolic calcium in rat hippocampal neurons. ${ }^{53,54}$ Due to the presence of the infusion cannula we were unable to use the FST to measure depressive-like behaviors. We therefore used the open field test to measure anxiety following TG infusion. Rats were placed into the open field arena $40 \mathrm{~min}$ after bilateral infusion of either vehicle or TG (Figures $5 \mathrm{a}$ and b). Locomotor activity and anxiety-related behaviors (for example, center square entries) were measured for $10 \mathrm{~min}$. Rats infused with TG exhibited lower locomotor activity and decreased center square entries indicating anxiogenic-like behavior compared to vehicle-infused rats (Figures $5 \mathrm{c}-\mathrm{e}$ ). Dorsal hippocampal slices were prepared immediately after completion of the open field test and somatic whole-cell current-clamp recordings were made from CA1 neurons. CA1 neurons from

Figure 4. Lentiviral-mediated knockdown of HCN1 protein expression in dorsal CA1 region/neurons led to resiliency to CUS. (a) Timeline of CUS, behavioral tests, and electrophysiology. (b) Body weight changes during CUS. (c) Representative coronal sections of the brains display the areas (CA1 region) infected by lentivirus. (d) Representative video tracking images during the last 5 min of open field test of age-matched individual rats exposed to CUS or CUS-shRNA-control or shRNA-HCN1 infusion. CUS or CUS-shRNA-control rats showed decreases in number of line crossing (e) and center square entries (f) compared to the control group. CUS-shRNA-HCN1 rats displayed increases in number of line crossing (e) and center square entries (f) compared to the CUS or CUS-shRNA-control groups. (g) CUS or CUS-shRNA-control rats showed a decreased sucrose preference (i.e., anhedonic-like effect) compared to the control group. CUS-shRNA-HCN1 rats showed an increased sucrose preference compared to the CUS or CUS-shRNA-control groups. (h) CUS or CUS-shRNA-control rats displayed an increase in passive activity (i.e., behavioral despair) compared to the control group. CUS-shRNA-HCN1 rats showed a decrease in passive activity compared to the CUS or CUS-shRNA-control groups. (I) Representative voltage responses with step current injections at $-73 \mathrm{mV}$ (750 ms). (j) $R_{\text {in }}$ was significantly reduced in the CUS or CUS-shRNA-control groups compared to the control group. When HCN1 protein was reduced, $\mathrm{R}_{\text {in }}$ was significantly increased compared to the CUS or CUS-shRNA-control groups. (k) Representative voltage traces and current injections at - 73 mV. (I) The profile of impedance amplitude for voltage traces in k. Vertical lines indicate the resonance frequencies. (m) Resonance frequency was significantly increased in the CUS or CUS-shRNA-control groups compared to the control group. CUS-induced an increase in $f_{R}$, which was significantly decreased by shRNA-HCN1 compared to the CUS or CUS-shRNA-control groups. Data are expressed as mean \pm s.e.m. with significance indicated by ${ }^{*} P<0.05$, compared with control group or CUS-shRNA-HCN1 group. CUS, chronic unpredictable stress. 
TG-treated rats had a depolarized RMP compared to CA1 neurons from vehicle-treated rats (Supplementary Table S7). At $-73 \mathrm{mV}$, dorsal CA1 neurons from TG-treated rats had a lower $R_{\text {in }}$ (Figures $5 f, g$ and Supplementary Table S7) and higher $f_{R}$ (Figures 5h-j and Supplementary Table S7) compared to neurons from vehicle-treated rats. Furthermore, $I_{h}$-dependent measures (for example, $R_{\text {in }}$ and $f_{\mathrm{R}}$ ) at different membrane potentials were consistent with an increase in $I_{\mathrm{h}}$ following treatment with TG (Figures $5 \mathrm{k}-\mathrm{n}$ and Supplementary Table S7). These results suggest that in vivo block of the SERCA pumps in the dorsal CA1 region a

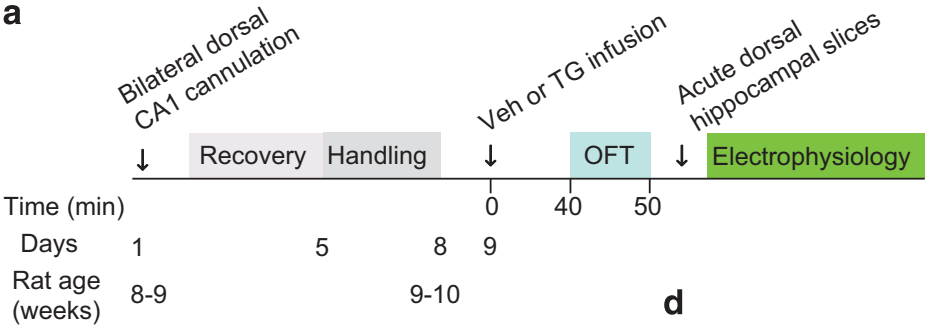

b

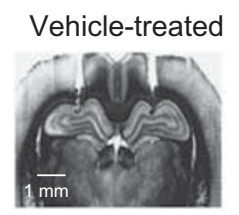

Thapsigargin-treated
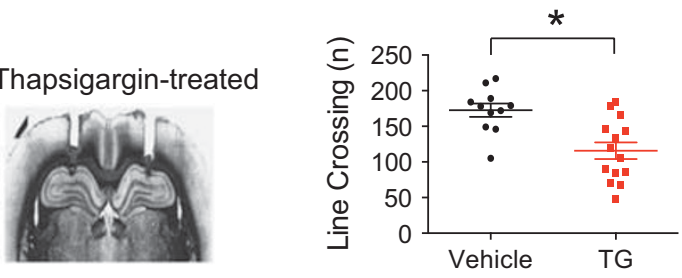

f
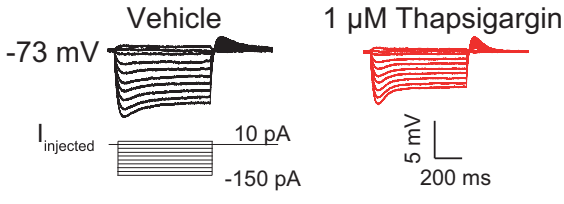

g

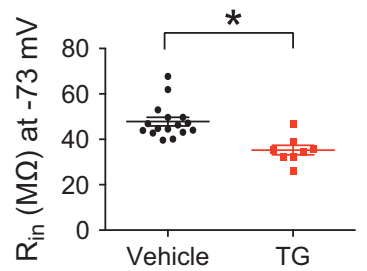

k
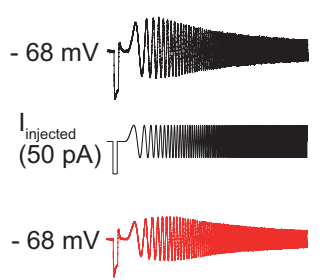

$\mathrm{I}_{\text {injected }}$
$(50 \mathrm{pA})$

I
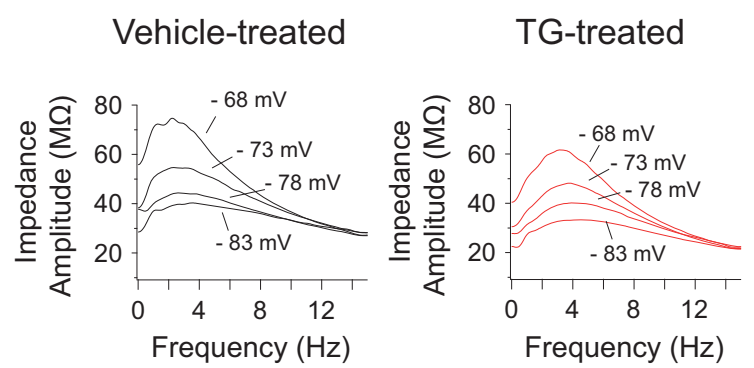

h

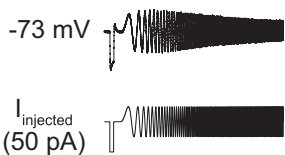

i

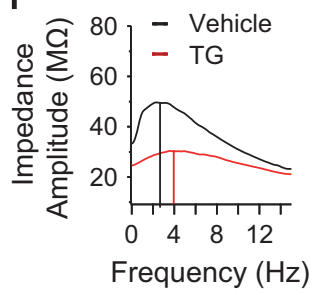

Vehicle-treated
$-73 \mathrm{mV}-\mathrm{Yu}$

$1 \mu \mathrm{M}$ thapsigargin-treated
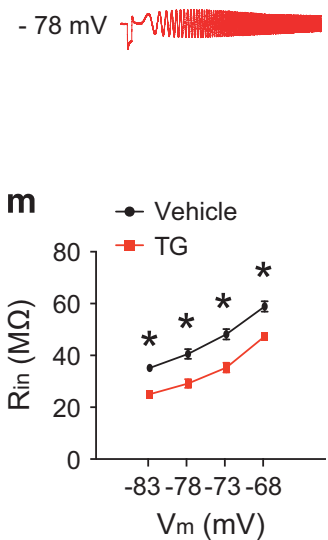

C

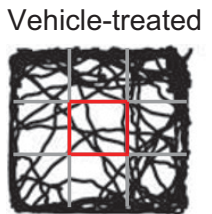

TG-treated

e

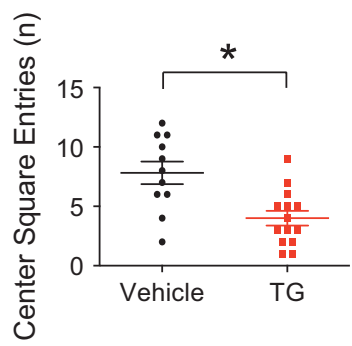

$1 \mu \mathrm{M}$ Thapsigargin
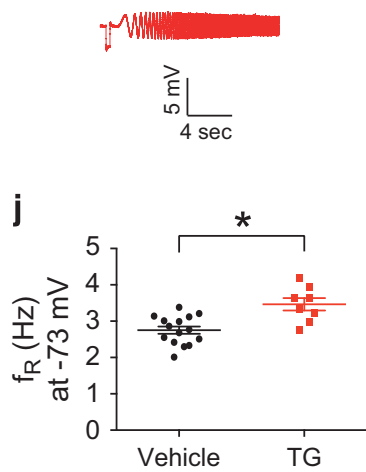

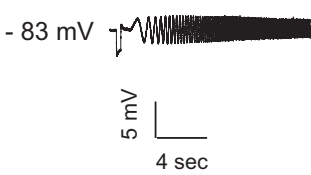

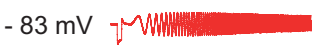

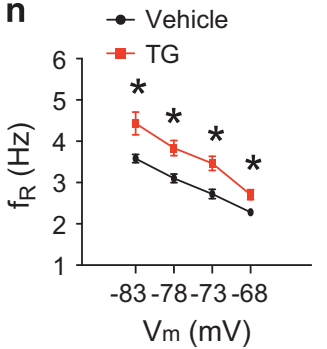


produced anxiogenic-like behavior and elevated perisomatic $I_{\mathrm{h}}$ similar to that observed in rats following CUS.

\section{DISCUSSION}

The major findings from our studies are that chronic, but not acute, stress resulted in increases in perisomatic HCN1 channels and functional $I_{\mathrm{h}}$ that correlates in time with the development of depression-like symptoms in rats. Furthermore, these changes in $I_{\mathrm{h}}$ were restricted to the dorsal region of the hippocampus. Previous reports from our laboratory and others support the hypothesis that reduction of functional $I_{\mathrm{h}}$ in the hippocampus produces antidepressant-like effects in normal rats/mice. Knockout of the subunits for h-channels (TRIP8b, HCN1 and HCN2) in mice resulted in a reduction of functional $I_{\mathrm{h}}$ and antidepressant-like behaviors. ${ }^{36}$ Furthermore, lentiviral-mediated knockdown of HCN1 protein expression in the dorsal CA1 region showed anxiolytic- and antidepressant-like effects. ${ }^{16}$ Previous reports suggested that the ventral hippocampus is heavily involved in emotion-related behaviors such as anxiety and fear responses. ${ }^{55,56}$ However, we found that despite the expected reduction in functional $I_{h}$, knockdown of HCN1 protein expression in the ventral CA1 region had no anxiolytic- or antidepressant-like effects. In agreement with these findings, we found CUS-dependent effects in h-channel expression and function in the dorsal but not ventral CA1 region. These findings suggest that there are brain region-specific changes in $I_{\mathrm{h}}$ in depression and perhaps in the action of antidepressants. Supporting this hypothesis, it has been reported that BDNF expression provides opposing effects in hippocampus and ventral tegmental area (VTA). A reduction of BDNF expression in hippocampus induces depressive-like behavior, ${ }^{57}$ whereas decreased BDNF expression in VTA leads to an antidepressant-like behavior. ${ }^{58}$ Chronic stress causes a reduction of BDNF expression in hippocampus, ${ }^{59}$ but an increase in BDNF expression in the VTA. ${ }^{60}$ In the hippocampus, BDNF protein levels are higher in the dorsal hippocampus compared to the ventral hippocampus. ${ }^{61}$ In agreement with our findings, 4 weeks of chronic mild stress significantly reduced BDNF protein expression in dorsal, but not in ventral hippocampus. ${ }^{61}$

The HCN1 subunit of h-channels is highly expressed in the hippocampal CA1 region and shows a gradient of increasing channel expression along the somatodendritic compartment. ${ }^{27-29}$ This distinct expression pattern of $I_{\mathrm{h}}$ contributes to the intrinsic membrane properties and synaptic integration in CA1 neurons. $^{30,31}$ Downregulation of $I_{h}$ and redistribution of HCN1 protein expression along the somatodendritic compartment have been implicated in acquired epilepsy. ${ }^{62-66}$ In an animal model of epilepsy, the gating of HCN channels was reduced by altered phosphorylation via calcineurin and p38 mitogen-activated protein kinase (p38 MAPK). ${ }^{67}$ Interestingly, HCN1 protein was redistributed from distal dendrites to the soma of CA1 neurons following status epilepticus. ${ }^{65}$ In the CUS model of depression, however, we observed only a perisomatic increase in HCN1 protein expression and $I_{\mathrm{h}}$ rather than the redistribution found in models of epilepsy. Furthermore, this perisomatic increase was only in dorsal hippocampus. Whether or not there are dorsal vs ventral differences in $I_{\mathrm{h}}$ expression in epilepsy models is not known.

Immunohistochemical staining with antibodies against HCN1 and HCN2 channels showed perisomatic increases in the expression of HCN1 and HCN2 (data not shown) protein in dorsal but not ventral CA1 region of hippocampus. In agreement with these results, somatic and dendritic whole-cell current-clamp recordings revealed that $I_{\mathrm{h}}$-related changes occurred in perisomatic, but not in dendritic regions of the CA1 neurons of the dorsal hippocampus following CUS. Because we observed an upregulation of perisomatic $I_{\mathrm{h}}$ in dorsal CA1 neurons following CUS, we examined whether this increased perisomatic $I_{\mathrm{h}}$ was a consequence of CUS. The time course of CUS experiments revealed that both depressive symptoms and increased perisomatic $I_{\mathrm{h}}$ in dorsal CA1 neurons occurred after 20-21 days CUS. These CUS-induced behavioral deficits and upregulation of perisomatic $I_{\mathrm{h}}$ were no longer observed in the recovery group, suggesting that abnormal subcellular expression of $I_{h}$ was dependent on the continual exposure to the CUS. Given that upregulation of $I_{h}$ is restricted to the perisomatic dorsal CA1 region following CUS, the reduced $R_{\text {in }}$ contributed, in part, to the reduction of neuronal excitability. We cannot, however, rule out the involvement of other channels. It is worth noting that McDermott et al., reported that 3-days of sleep deprivation, which may have a stress component, produced a reduction of neuronal excitability and input resistance in hippocampal CA1 neurons and speculated that this could be due in part to increases in $I_{\mathrm{h}}$ as well as changes in several additional channels. ${ }^{68}$ Importantly, our acute stress experiments demonstrated that rats subjected to a single 30-min restraint stress or 2 days of forced swim testing displayed no significant changes in electrophysiological properties in dorsal CA1 neurons. Consistent with our results, a single episode of restraint stress has no effect on neuronal excitability in lateral amygdala neurons. ${ }^{69}$ These results suggest that increased perisomatic $I_{\mathrm{h}}$ was in response to repeated stress exposure and not by acute stress. Recently, Han et al., $^{17}$ reported that restoring TRIP8b expression in the dorsal CA1 region of TRIP8b knockout mice led to enhancement of $\mathrm{HCN}$ protein expression and reversed the antidepressant-like behaviors in TRIP8b knockout mice. In agreement, we also found that reduced HCN1 protein expression along the somatodendritic region of dorsal CA1 region in CUS-shRNAHCN1 rats produced resiliency to CUS.

Persistent elevated cortisol is frequently observed in patients with major depressive disorder. ${ }^{19,70}$ Patients with depression exhibit abnormal increases in intracellular calcium levels in platelets and lymphocytes. ${ }^{50,51}$ Chronic stress induces hypersecretion of glucocorticoids, resulting in an increase in calcium influx via L-type voltage-gated $\mathrm{Ca}^{2+}$ channels. ${ }^{48,49,71}$ Previous reports from our laboratory showed that depletion of intracellular calcium stores induces a perisomatic increase in $I_{\mathrm{h}}$ in CA1 neurons. ${ }^{37,39}$ This perisomatic increase in $I_{h}$ is mediated in part by a rise in intracellular calcium and activation of inositol 1,4,5 trisphosphate receptors (InsP $\mathrm{P}_{3} \mathrm{Rs}$ ) and store operated channels (SOCs). ${ }^{37,52} \mathrm{We}$

Figure 5. In vivo block of SERCA pumps in dorsal CA1 region/neurons produced an anxiogenic-like behavior and increased $I_{\mathrm{h}}$. (a) Timeline of thapsigargin experiment. (b) Representative coronal sections of the brains display the location of the infusion needle track within the dorsal hippocampus of CA1. (c) Representative video tracking images during the $10 \mathrm{~min}$ of open field test of age-matched individual rats with vehicle or thapsigargin infusion. Rats bilaterally infused with $1 \mu \mathrm{m}$ thapsigargin showed a decreased locomotor activity (d) and lowered center square entries (e) compared to the vehicle-treated group. (f) Representative voltage responses with step current injections at $-73 \mathrm{mV}$ $(750 \mathrm{~ms})$. (g) TG-treated dorsal CA1 neurons had a decreased $\mathrm{R}_{\text {in }}$ compared to vehicle-treated group. (h) Representative voltage traces and current injections at $-73 \mathrm{mV}$. (i) The profile of impedance amplitude for voltage traces in (h). Vertical lines indicate the resonance frequencies. (j) TG-treated dorsal CA1 neurons showed an increased $f_{\mathrm{R}}$ compared to vehicle-treated group. (k) Representative voltage traces and current injections at different membrane potential (ranging from $-68 \mathrm{mV}$ to $-83 \mathrm{mV} ;-5 \mathrm{mV}$ interval). (I) The profile of impedance amplitude for voltage traces in (k). TG-treated dorsal CA1 neurons displayed a lowered $R_{\text {in }}$ ( $\mathbf{k}$ and $\mathbf{m}$ ) and a higher $f_{\mathrm{R}}(\mathbf{k}$ and $\mathbf{n})$ compared to vehicle-treated group. Data are expressed as mean \pm s.e.m. with significance indicated by ${ }^{*} P<0.05$, compared with vehicle-treated group. OFT, open field test; TG, thapsigargin. 
found that bilateral infusion of thapsigargin to block SERCA pumps and deplete intracellular calcium stores produced anxiogenic-like behavior and increased perisomatic $I_{\mathrm{h}}$. Therefore one possible mechanism underlying the depressive phenotype following CUS is the release of calcium from intracellular stores, activation of $\operatorname{InsP}_{3} \mathrm{Rs}$ and $\mathrm{SOCs}$, and a resulting upregulation of perisomatic $I_{\mathrm{h}}$. Supporting this hypothesis, in vitro cyclopiazonic acid or thapsigargin treatment for $10 \mathrm{~min}$ led to a persistent change in $I_{\mathrm{h}}$ plasticity (for example, decreased $\mathrm{R}_{\text {in }}$ and increased $f_{\mathrm{R}}$ ) of hippocampal CA1 neurons over at least $70 \mathrm{~min}^{37,39}$ Furthermore, given that the CUS-induced upregulation of $I_{\mathrm{h}}$ and increase in HCN1 protein expression were observed at least an hour to a few days following behavioral tests, this could be due to a persistent effect by the activation of InsP $_{3} \mathrm{Rs}$ and SOCs.

Patients with major depression are prone to develop temporal lobe epilepsy (TLE). ${ }^{72,73}$ Because a bidirectional relationship between major depression and TLE has been reported, ${ }^{73}$ these two disorders might share cellular pathological mechanisms. In our study, we found upregulation of $I_{\mathrm{h}}$ in the dorsal perisomatic CA1 region of hippocampus in the CUS model of depression. Downregulatioin of $I_{\mathrm{h}}$ in the dendritic CA1 region of hippocampus has been reported in the kainic acid or pilocarpine model of TLE. ${ }^{65,67}$ In agreement, lamotrigine, an antiepileptic drug, is known to increase dendritic $I_{\mathrm{h}}$ in hippocampal CA1 neurons. ${ }^{74}$ Thus, the subcellular distribution and expression of $I_{\mathrm{h}}$ in the CA1 neurons along the dorsal-ventral hippocampus might contribute to the cellular and molecular mechanisms of these two disorders-major depression and TLE.

In summary, we found brain and subcellular region-specific changes in the expression of hippocampal $I_{\mathrm{h}}$ in the CUS model of depression. This upregulation of perisomatic $I_{h}$ could be associated with increased intracellular calcium levels in the dorsal CA1 region. Furthermore, reduction of $\mathrm{HCN} 1$ protein expression is sufficient to provide resiliency to CUS. In combination with previous reports, these results suggest that therapeutic interventions targeting $\mathrm{h}$-channels and their regulatory mechanisms are a promising strategy in the treatment of major depressive disorder.

\section{CONFLICT OF INTEREST}

The authors declare no conflict of interest.

\section{ACKNOWLEDGMENTS}

This work was supported by grants from National Institutes of Health grants MH48432 (D.J.) and MH100510 (D.H.B.) and a Brain and Behavior Research Foundation Young Investigator award (\#22745) from John Kennedy Harrison to Chung Sub Kim. We thank all members of Johnston laboratory for reviewing and comments throughout this study, to Komal Himanshu Parikh for performing neuronal reconstruction, Siddharth Das for western blotting, and Dr Payne Y. Chang for behavioral software. We also thank to Drs Ryuichi Shigemoto for HCN1 and HCN2 antibodies and Paul Pfaffinger for the lentiviral vectors.

\section{REFERENCES}

1 Campbell S, Marriott M, Nahmias C, MacQueen GM. Lower hippocampal volume in patients suffering from depression: a meta-analysis. Am J Psychiatry 2004; 161: 598-607.

2 Kennedy SH, Evans KR, Kruger S, Mayberg HS, Meyer JH, McCann S et al. Changes in regional brain glucose metabolism measured with positron emission tomography after paroxetine treatment of major depression. Am J Psychiatry 2001; 158: 899-905.

3 Mayberg HS, Brannan SK, Tekell JL, Silva JA, Mahurin RK, McGinnis S et al. Regional metabolic effects of fluoxetine in major depression: serial changes and relationship to clinical response. Biol Psychiatry 2000; 48: 830-843.

4 Santarelli L, Saxe M, Gross C, Surget A, Battaglia F, Dulawa S et al. Requirement of hippocampal neurogenesis for the behavioral effects of antidepressants. Science 2003; 301: 805-809.

5 Nestler EJ, Barrot M, DiLeone RJ, Eisch AJ, Gold SJ, Monteggia LM. Neurobiology of depression. Neuron 2002; 34: 13-25.
6 McEwen BS. Stress and hippocampal plasticity. Annu Rev Neurosci 1999; 22: 105-122.

7 Moser MB, Moser El. Functional differentiation in the hippocampus. Hippocampus 1998; 8: 608-619.

8 Dong HW, Swanson LW, Chen L, Fanselow MS, Toga AW. Genomic-anatomic evidence for distinct functional domains in hippocampal field CA1. Proc Natl Acad Sci USA 2009; 106: 11794-11799.

9 Fanselow MS, Dong HW. Are the dorsal and ventral hippocampus functionally distinct structures? Neuron 2010; 65: 7-19.

10 Maller JJ, Daskalakis ZJ, Fitzgerald PB. Hippocampal volumetrics in depression: the importance of the posterior tail. Hippocampus 2007; 17: 1023-1027.

11 Higuchi F, Uchida S, Yamagata H, Abe-Higuchi N, Hobara T, Hara K et al. Hippocampal MicroRNA-124 Enhances Chronic Stress Resilience in Mice. J Neurosci 2016; 36: 7253-7267.

12 Taliaz D, Loya A, Gersner R, Haramati S, Chen A, Zangen A. Resilience to chronic stress is mediated by hippocampal brain-derived neurotrophic factor. $J$ Neurosci 2011; 31: 4475-4483.

13 Lu XY, Kim CS, Frazer A, Zhang W. Leptin: a potential novel antidepressant. Proc Natl Acad Sci USA 2006; 103: 1593-1598.

14 McLaughlin RJ, Hill MN, Morrish AC, Gorzalka BB. Local enhancement of cannabinoid CB1 receptor signalling in the dorsal hippocampus elicits an antidepressant-like effect. Behav Pharmacol 2007; 18: 431-438.

15 Padovan CM, Guimaraes FS. Antidepressant-like effects of NMDA-receptor antagonist injected into the dorsal hippocampus of rats. Pharmacol Biochem Behav 2004; 77: 15-19.

16 Kim CS, Chang PY, Johnston D. Enhancement of dorsal hippocampal activity by knockdown of HCN1 channels leads to anxiolytic- and antidepressant-like behaviors. Neuron 2012; 75: 503-516.

17 Han Y, Heuermann RJ, Lyman KA, Fisher D, Ismail QA, Chetkovich DM. HCN-channel dendritic targeting requires bipartite interaction with TRIP8b and regulates antidepressant-like behavioral effects. Mol Psychiatry 2017; 22: 458-465.

18 Kendler KS, Karkowski LM, Prescott CA. Causal relationship between stressful life events and the onset of major depression. Am J Psychiatry 1999; 156: 837-841.

19 Sachar EJ, Hellman L, Fukushima DK, Gallagher TF. Cortisol production in depressive illness. A clinical and biochemical clarification. Arch Gen Psychiatry 1970; 23: 289-298.

20 Carroll BJ, Schroeder K, Mukhopadhyay S, Greden JF, Feinberg M, Ritchie J et al. Plasma dexamethasone concentrations and cortisol suppression response in patients with endogenous depression. J Clin Endocrinol Metab 1980; 51: 433-437.

21 Willner P. Chronic mild stress (CMS) revisited: consistency and behaviouralneurobiological concordance in the effects of CMS. Neuropsychobiology 2005; 52: 90-110.

22 Katz RJ. Animal models and human depressive disorders. Neurosci Biobehav Rev 1981; 5: 231-246.

23 Willner P. The validity of animal models of depression. Psychopharmacology (Berl) 1984; 83: 1-16.

24 Katz RJ. Animal model of depression: pharmacological sensitivity of a hedonic deficit. Pharmacol Biochem Behav 1982; 16: 965-968.

25 Willner P, Towell A, Sampson D, Sophokleous S, Muscat R. Reduction of sucrose preference by chronic unpredictable mild stress, and its restoration by a tricyclic antidepressant. Psychopharmacology (Berl) 1987; 93: 358-364.

26 Monteggia LM, Eisch AJ, Tang MD, Kaczmarek LK, Nestler EJ. Cloning and localization of the hyperpolarization-activated cyclic nucleotide-gated channel family in rat brain. Brain Res Mol Brain Res 2000; 81: 129-139.

27 Lorincz A, Notomi T, Tamas G, Shigemoto R, Nusser Z. Polarized and compartment-dependent distribution of HCN1 in pyramidal cell dendrites. Nat Neurosci 2002; 5: 1185-1193.

28 Magee JC. Dendritic hyperpolarization-activated currents modify the integrative properties of hippocampal CA1 pyramidal neurons. J Neurosci 1998; 18: 7613-7624.

29 Notomi T, Shigemoto R. Immunohistochemical localization of Ih channel subunits, HCN1-4, in the rat brain. J Comp Neurol 2004; 471: 241-276.

30 Magee JC. Dendritic Ih normalizes temporal summation in hippocampal CA1 neurons. Nat Neurosci 1999; 2: 508-514.

31 Pape HC. Queer current and pacemaker: the hyperpolarization-activated cation current in neurons. Annu Rev Physiol 1996; 58: 299-327.

32 Shah MM, Anderson AE, Leung V, Lin X, Johnston D. Seizure-induced plasticity of $h$ channels in entorhinal cortical layer III pyramidal neurons. Neuron 2004; 44: 495-508.

$33 \mathrm{Hu} \mathrm{H}$, Vervaeke K, Storm JF. Two forms of electrical resonance at theta frequencies, generated by $\mathrm{M}$-current, $\mathrm{h}$-current and persistent $\mathrm{Na}^{+}$current in rat hippocampal pyramidal cells. J Physiol 2002; 545(Pt 3): 783-805.

34 Narayanan $R$, Johnston $D$. Long-term potentiation in rat hippocampal neurons is accompanied by spatially widespread changes in intrinsic oscillatory dynamics and excitability. Neuron 2007; 56: 1061-1075. 
35 Cao JL, Covington HE 3rd, Friedman AK, Wilkinson MB, Walsh JJ, Cooper DC et al. Mesolimbic dopamine neurons in the brain reward circuit mediate susceptibility to social defeat and antidepressant action. J Neurosci 2010; 30: 16453-16458.

36 Lewis AS, Vaidya SP, Blaiss CA, Liu Z, Stoub TR, Brager DH et al. Deletion of the Hyperpolarization-Activated Cyclic Nucleotide-Gated Channel Auxiliary Subunit TRIP8b Impairs Hippocampal Ih Localization and Function and Promotes Antidepressant Behavior in Mice. J Neurosci 2011; 31: 7424-7440.

37 Narayanan R, Dougherty KJ, Johnston D. Calcium store depletion induces persistent perisomatic increases in the functional density of $h$ channels in hippocampal pyramidal neurons. Neuron 2010; 68: 921-935.

38 Prakriya M, Lewis RS. Store-Operated Calcium Channels. Physiol Rev 2015; 95 1383-1436.

39 Clemens AM, Johnston D. Age- and location-dependent differences in store depletion-induced h-channel plasticity in hippocampal pyramidal neurons. J Neurophysiol 2014; 111: 1369-1382.

40 Lucki I. The forced swimming test as a model for core and component behavioral effects of antidepressant drugs. Behav Pharmacol 1997; 8: 523-532.

$41 \mathrm{Kim}$ CS, Johnston D. A1 adenosine receptor-mediated GIRK channels contribute to the resting conductance of CA1 neurons in the dorsal hippocampus. J Neurophysiol 2015; 113: 2511-2523.

42 Stuart GJ, Dodt HU, Sakmann B. Patch-clamp recordings from the soma and dendrites of neurons in brain slices using infrared video microscopy. Pflugers Arch 1993; 423: 511-518.

43 Dougherty KA, Islam T, Johnston D. Intrinsic excitability of CA1 pyramidal neurones from the rat dorsal and ventral hippocampus. J Physiol 2012; 590(Pt 22): 5707-5722.

44 Altar CA. Neurotrophins and depression. Trends Pharmacol Sci 1999; 20: 59-61.

45 Henriksen EJ, Colgin LL, Barnes CA, Witter MP, Moser MB, Moser El. Spatial representation along the proximodistal axis of CA1. Neuron 2010; 68: 127-137.

46 Malik R, Dougherty KA, Parikh K, Byrne C, Johnston D. Mapping the electrophysiological and morphological properties of CA1 pyramidal neurons along the longitudinal hippocampal axis. Hippocampus 2016; 26: 341-361.

47 Routh BN, Johnston D, Harris K, Chitwood RA. Anatomical and electrophysiological comparison of CA1 pyramidal neurons of the rat and mouse. J Neurophysiol 2009; 102: 2288-2302.

48 Kerr DS, Campbell LW, Thibault O, Landfield PW. Hippocampal glucocorticoid receptor activation enhances voltage-dependent $\mathrm{Ca} 2+$ conductances: relevance to brain aging. Proc Natl Acad Sci USA 1992; 89: 8527-8531.

49 Karst H, Karten YJ, Reichardt HM, de Kloet ER, Schutz G, Joels M. Corticosteroid actions in hippocampus require DNA binding of glucocorticoid receptor homodimers. Nat Neurosci 2000; 3: 977-978.

50 Dubovsky SL, Murphy J, Thomas M, Rademacher J. Abnormal intracellular calcium ion concentration in platelets and lymphocytes of bipolar patients. Am $J$ Psychiatry 1992; 149: 118-120.

51 Emamghoreishi M, Schlichter L, Li PP, Parikh S, Sen J, Kamble A et al. High intracellular calcium concentrations in transformed lymphoblasts from subjects with bipolar I disorder. Am J Psychiatry 1997; 154: 976-982.

52 Ashhad S, Johnston D, Narayanan R. Activation of InsP(3) receptors is sufficient for inducing graded intrinsic plasticity in rat hippocampal pyramidal neurons. J Neurophysiol 2015; 113: 2002-2013.

53 Harvey J, Collingridge GL. Thapsigargin blocks the induction of long-term potentiation in rat hippocampal slices. Neurosci Lett 1992; 139: 197-200.

54 Wang Y, Mattson MP, Furukawa K. Endoplasmic reticulum calcium release is modulated by actin polymerization. J Neurochem 2002; 82: 945-952.

55 Burns LH, Annett L, Kelley AE, Everitt BJ, Robbins TW. Effects of lesions to amygdala, ventral subiculum, medial prefrontal cortex, and nucleus accumbens on the reaction to novelty: implication for limbic-striatal interactions. Behav Neurosci 1996; 110: 60-73.

56 Kjelstrup KG, Tuvnes FA, Steffenach HA, Murison R, Moser El, Moser MB. Reduced fear expression after lesions of the ventral hippocampus. Proc Natl Acad Sci USA 2002; 99: 10825-10830.
57 Taliaz D, Stall N, Dar DE, Zangen A. Knockdown of brain-derived neurotrophic factor in specific brain sites precipitates behaviors associated with depression and reduces neurogenesis. Mol Psychiatry 2010; 15: 80-92.

58 Berton O, McClung CA, Dileone RJ, Krishnan V, Renthal W, Russo SJ et al. Essential role of BDNF in the mesolimbic dopamine pathway in social defeat stress. Science 2006; 311: 864-868.

59 Gronli J, Bramham C, Murison R, Kanhema T, Fiske E, Bjorvatn B et al. Chronic mild stress inhibits BDNF protein expression and CREB activation in the dentate gyrus but not in the hippocampus proper. Pharmacol Biochem Behav 2006; 85: 842-849.

60 Fanous S, Hammer RP Jr, Nikulina EM. Short- and long-term effects of intermittent social defeat stress on brain-derived neurotrophic factor expression in mesocorticolimbic brain regions. Neuroscience 2010; 167: 598-607.

61 Toth E, Gersner R, Wilf-Yarkoni A, Raizel H, Dar DE, Richter-Levin G et al. Age-dependent effects of chronic stress on brain plasticity and depressive behavior. J Neurochem 2008; 107: 522-532.

62 Ludwig A, Budde T, Stieber J, Moosmang S, Wahl C, Holthoff K et al. Absence epilepsy and sinus dysrhythmia in mice lacking the pacemaker channel HCN2. EMBO J 2003; 22: 216-224.

63 Huang Z, Walker MC, Shah MM. Loss of dendritic HCN1 subunits enhances cortical excitability and epileptogenesis. J Neurosci 2009; 29: 10979-10988.

64 Santoro B, Lee JY, Englot DJ, Gildersleeve S, Piskorowski RA, Siegelbaum SA et al. Increased seizure severity and seizure-related death in mice lacking HCN1 channels. Epilepsia 2010; 51: 1624-1627.

65 Shin M, Brager D, Jaramillo TC, Johnston D, Chetkovich DM. Mislocalization of $h$ channel subunits underlies h channelopathy in temporal lobe epilepsy. Neurobiol Dis 2008; 32: 26-36.

66 Jung S, Jones TD, Lugo JN Jr, Sheerin AH, Miller JW, D'Ambrosio R et al. Progressive dendritic $\mathrm{HCN}$ channelopathy during epileptogenesis in the rat pilocarpine model of epilepsy. J Neurosci 2007; 27: 13012-13021.

67 Jung S, Bullis JB, Lau IH, Jones TD, Warner LN, Poolos NP. Downregulation of dendritic $\mathrm{HCN}$ channel gating in epilepsy is mediated by altered phosphorylation signaling. J Neurosci 2010; 30: 6678-6688.

68 McDermott CM, LaHoste GJ, Chen C, Musto A, Bazan NG, Magee JC. Sleep deprivation causes behavioral, synaptic, and membrane excitability alterations in hippocampal neurons. J Neurosci 2003; 23: 9687-9695.

69 Rosenkranz JA, Venheim ER, Padival M. Chronic stress causes amygdala hyperexcitability in rodents. Biol Psychiatry 2010; 67: 1128-1136.

70 Gibbons JL, Mc HP. Plasma cortisol in depressive illness. J Psychiatr Res 1962; 1: 162-171.

71 Zhao Y, Xu J, Gong J, Qian L. L-type calcium channel current up-regulation by chronic stress is associated with increased alpha(1c) subunit expression in rat ventricular myocytes. Cell Stress Chaperones 2009; 14: 33-41.

72 Hesdorffer DC, Hauser WA, Annegers JF, Cascino G. Major depression is a risk factor for seizures in older adults. Ann Neurol 2000; 47: 246-249.

73 Kanner AM, Balabanov A. Depression and epilepsy: how closely related are they? Neurology 2002; 58(8 Suppl 5): S27-S39.

74 Poolos NP, Migliore M, Johnston D. Pharmacological upregulation of h-channels reduces the excitability of pyramidal neuron dendrites. Nat Neurosci 2002; 5: 767-774.

(c) (1) $\Theta$ This work is licensed under a Creative Commons AttributionNonCommercial-NoDerivs 4.0 International License. The images or other third party material in this article are included in the article's Creative Commons license, unless indicated otherwise in the credit line; if the material is not included under the Creative Commons license, users will need to obtain permission from the license holder to reproduce the material. To view a copy of this license, visit http:// creativecommons.org/licenses/by-nc-nd/4.0/

(c) The Author(s) 2018

Supplementary Information accompanies the paper on the Molecular Psychiatry website (http://www.nature.com/mp) 